\title{
Multiresolutional Techniques in Finite Element Method Solution of Eigenvalue Problem
}

\author{
Marcin Kamiński \\ Chair of Mechanics of Materials, Technical University of Łódź \\ Al. Politechniki 6, 93-590 Łodz, POLAND, tel/fax 48-42-6313551 \\ marcin@kmm-1x.p.lodz.pl, marcinka@p.lodz.pl
}

\begin{abstract}
Computational analysis of unidirectional transient problems in multiscale heterogeneous media using specially adopted homogenization technique and the Finite Element Method is described below. Multiresolutional homogenization being the extension of the classical micro-macro traditional approach is used to calculate effective parameters of the composite. Effectiveness of the method is compared against previous techniques thanks to the FEM solution of some engineering problems with real material parameters and with their homogenized values. Further computational studies are necessary in this area, however application of the multiresolutional technique is justified by the natural multiscale character of composites.
\end{abstract}

\section{Introduction}

Wavelet analysis [1] perfectly reflects the very demanding needs of composite materials computational modeling. It is due to the fact that wavelet functions like Haar, sinusoidal (harmonic), Gabor, Morlet or Daubechies, for instance, relating neighboring scales in the medium analysed can efficiently model a variety of heterogeneities preserving composites periodicity, for instance. It is evident now that wavelet techniques may serve for analysis in the finest scale by various numerical techniques $[2,4,5]$ as well as using multiresolutional analysis (MRA) $[3,5,6,8]$. The first method leads to the exponential increase of the total number of degrees of freedom in the model, because each new decomposition level almost doubles this number, while an application of the homogenization method is connected with determination of effective material parameters.

Both methodologies are compared here in the context of eigenvalue problem solution for a simply supported linear elastic Euler-Bernoulli beam using the Finite Element Method (FEM) computational procedures. The corresponding comparison made for a transient heat transfer has been discussed before in [5]. Homogenization of a composite is performed here through (1) simple spatial averaging of composite properties, (2) two-scale classical approach [7] as well as (3) thanks to the multiresolutional technique based on the Haar wavelets. An application of the symbolic package MAPLE guarantees an efficient integration of algebraic formulas defining effective properties for a composite with material properties given by some wavelet functions. 


\section{Multiresolutional Homogenization Scheme}

MRA approach uses the algebraic transformation between various scales provided by the wavelet analysis to determine the fine-scale behavior and introduce it explicitly into the macroscopic equilibrium equations. The following relation:

$$
\Omega_{0} \subset \Omega_{-1} \subset \Omega_{-2} \subset \ldots
$$

defines the hierarchical geometry of the scales and this chain of subspaces is so defined that $\Omega_{\mathrm{j}}$ is "finer" than $\Omega_{\mathrm{j}+1}$. Further, let us note that the main assumption on general homogenization procedure for transient problems is a separate averaging of the coefficients from the governing partial differential equation responsible for a static behavior and of the unsteady component. The problem can be homogenized only if its equilibrium can be expressed by the following operator equation:

$$
\mathrm{BT}+\mathrm{u}+\lambda=\mathrm{L}(\mathrm{AT}+\mathrm{v})
$$

This equation in the multiscale notation can be rewritten at the given scale $\mathrm{j}$ as

$$
\mathrm{B}_{0}^{(\mathrm{j})} \mathrm{T}_{0}^{(\mathrm{j})}+\mathrm{u}_{0}^{(\mathrm{j})}+\lambda=\mathrm{L}_{0}\left(\mathrm{~A}_{0}^{(\mathrm{j})} \mathrm{T}_{0}^{(\mathrm{j})}+\mathrm{v}_{0}^{(\mathrm{j})}\right),
$$

with the recurrence relations used $\mathrm{j}$ times to compute $\mathrm{B}_{0}^{(\mathrm{j})}, \mathrm{A}_{0}^{(\mathrm{j})}, \mathrm{u}_{0}^{(\mathrm{j})}, \mathrm{v}_{0}^{(\mathrm{j})}$. MRA homogenization theorem is obtained as a limit for $\mathrm{j} \rightarrow-\infty$

$$
\mathrm{B}_{0}^{(-\infty)} \mathrm{T}_{0}^{(-\infty)}+\mathrm{u}_{0}^{(-\infty)}+\lambda=\mathrm{L}_{0}\left(\mathrm{~A}_{0}^{(-\infty)} \mathrm{T}_{0}^{(-\infty)}+\mathrm{v}_{0}^{(-\infty)}\right)
$$

which enables to eliminate infinite number of the geometrical scales with the reduced coefficients $\mathrm{B}_{0}^{(-\infty)}, \mathrm{A}_{0}^{(-\infty)}$. If the limits defining the matrices $\mathrm{B}_{0}^{(-\infty)}$ and $\mathrm{A}_{0}^{(-\infty)}$ exist, then there exist constant matrices $\mathrm{B}^{\mathrm{h}}, \mathrm{A}^{\mathrm{h}}$ and forcing terms $\mathrm{u}^{\mathrm{h}}, \mathrm{v}^{\mathrm{h}}$, such that the reduced coefficients and forcing terms are given by $\mathrm{B}_{0}^{(-\infty)}, \mathrm{A}_{0}^{(-\infty)}, \mathrm{u}_{0}^{(-\infty)}, \mathrm{v}_{0}^{(-\infty)}$. The homogenized coefficients are equal to

$$
\begin{gathered}
A^{h}=A_{0}^{(-\infty)}, B^{h}=A^{h} \tilde{A}^{-1}-I, \\
u^{h}=u_{0}^{(-\infty)}+\left(I-\frac{1}{2} \tilde{A}-\tilde{A}\left(\exp (\tilde{A}-I)^{-1} A^{h}\right)^{-1} v^{h}\right),
\end{gathered}
$$

where

$$
\widetilde{\mathrm{A}}=\log \left(\mathrm{I}+\left(\mathrm{I}+\mathrm{B}_{0}^{(-\infty)}-\frac{1}{2} \mathrm{~A}^{\mathrm{h}}\right)^{-1} \mathrm{~A}^{\mathrm{h}}\right) .
$$

As the example let us review the static equilibrium of elastic Euler-Bernoulli beam

$$
-\frac{d}{d x}\left(E(x) \frac{d}{d x} u(x)\right)=f(x) ; x \in[0,1],
$$

where $\mathrm{E}(\mathrm{x})$, defining material properties of the heterogeneous medium, varies arbitrarily on many scales. The unit interval denotes here the Representative Volume Element (RVE), called also the periodicity cell. This equation can represent linear elastic behavior of unidirectional structures as well as unidirectional heat conduction and other related physical fields. A periodic structure with a small parameter $\varepsilon>0$, 
tending to 0 , relating the lengths of the periodicity cell and the entire composite, is considered in a classical approach. The displacements are expanded as

$$
u(x)=u^{(0)}(x, y)+\varepsilon^{1} u^{(1)}(x, y)+\varepsilon^{2} u^{(2)}(x, y)+\ldots,
$$

where $\mathrm{u}^{(\mathrm{i})}(\mathrm{x}, \mathrm{y})$ are also periodic; the coordinate $\mathrm{x}$ is introduced for macro scale, while $\mathrm{y}$ - in micro scale. Introducing these expansions into classical Hooke's law, the homogenized elastic modulus is obtained as [6]

$$
\mathrm{E}^{(\mathrm{eff})}=\left(\int_{\Omega} \frac{\mathrm{dy}}{\mathrm{E}(\mathrm{y})}\right)^{-1} \text {. }
$$

The method called multiresolutional starts from the following decomposition:

$$
\left\{\begin{array}{l}
\frac{d}{d x} u(x)=\frac{v(x)}{E(x)} \\
\frac{d}{d x} v(x)=-f(x)
\end{array}\right.
$$

to determine the homogenized coefficient $\mathrm{E}^{(\mathrm{eff})}$ constant for $\mathrm{x} \in[0,1]$. Therefore

$$
\left(\begin{array}{l}
u(x) \\
v(x)
\end{array}\right)-\left(\begin{array}{c}
u(0) \\
v(0)
\end{array}\right)=\int_{0}^{x}\left(\left(\begin{array}{cc}
0 & E(t)^{-1} \\
0 & 0
\end{array}\right)\left(\begin{array}{c}
u(t) \\
v(t)
\end{array}\right)+\left(\begin{array}{c}
0 \\
-f(t)
\end{array}\right)\right) d t .
$$

The reduction algorithm between multiple scales of the composite consists in determination of such effective tensors $B^{(\text {eff })}, A^{(\text {eff })}, p^{(\text {eff })}$ and $q^{(\text {eff })}$, such that

$$
\left(\mathbf{I}+\mathrm{B}^{(\text {eff })}\left(\begin{array}{c}
\mathrm{u}(\mathrm{x}) \\
\mathrm{v}(\mathrm{x})
\end{array}\right)+\mathrm{q}^{(\text {eff })}+\lambda=\int_{0}^{\mathrm{x}}\left(\mathrm{A}^{(\text {eff })}\left(\begin{array}{c}
\mathrm{u}(\mathrm{t}) \\
\mathrm{v}(\mathrm{t})
\end{array}\right)+\mathrm{p}^{(\text {eff })}\right) \mathrm{dt},\right.
$$

where $\mathbf{I}$ is an identity matrix. In our case we apply

$$
\mathrm{B}^{(\text {eff })}=\left(\begin{array}{ll}
0 & 0 \\
0 & 0
\end{array}\right) ; \mathrm{A}^{(\text {eff })}=\left(\begin{array}{cc}
0 & \mathrm{C}_{1}-2 \mathrm{C}_{2} \\
0 & 0
\end{array}\right), \quad \mathrm{C}_{1}=\int_{0}^{1} \frac{\mathrm{dt}}{\mathrm{E}(\mathrm{t})} ; \mathrm{C}_{2}=\int_{0}^{1} \frac{\left(\mathrm{t}-\frac{1}{2}\right) \mathrm{dt}}{\mathrm{E}(\mathrm{t})}
$$

Furthermore, for $\mathrm{f}(\mathrm{x})=0$ there holds $\mathrm{p}^{(\text {eff })}=\mathrm{q}^{(\text {eff })}=0$, while, in a general case, $\mathrm{B}^{(\text {eff })}$ and $\mathrm{A}^{\text {(eff) }}$ do not depend on $\mathrm{p}$ and $\mathrm{q}$.

\section{Multiresolutional Finite Element Method}

Let us consider the governing equation

$$
-\mathrm{e} \nabla^{2} \mathrm{u}=\mathrm{f}, \mathrm{x} \in \Omega
$$

with

$$
\mathrm{u}=0, \mathrm{x} \in \Gamma_{\mathrm{u}} \subset \partial \Omega .
$$

Variational formulation of this problem for the multiscale medium at the scale $\mathrm{k}$ is given as

$$
\int_{\Omega} \mathrm{e} \nabla \mathrm{u}_{\mathrm{k}} \nabla \varphi_{\mathrm{k}} \mathrm{d} \Omega+\int_{\Omega} \gamma \mathrm{u}_{\mathrm{k}} \varphi_{\mathrm{k}} \mathrm{d} \Omega=\int_{\Gamma} \mathrm{f} \varphi_{\mathrm{k}} \mathrm{d} \Gamma, \mathrm{x} \in \Omega
$$


Solution of the problem must be found recursively by using some transformation between the neighboring scales. Hence, the following nonsingular $\mathrm{n} \times \mathrm{n}$ wavelet transform matrix $\mathbf{W}_{\mathrm{k}}$ is introduced [2]:

$$
\mathbf{W}_{\mathrm{k}}=\mathbf{T}_{\mathrm{k}}\left[\begin{array}{cc}
\mathbf{T}_{\mathrm{k}-1} & 0 \\
0 & \mathbf{I}_{\mathrm{k}-1}
\end{array}\right],
$$

and

$$
\psi_{\mathrm{k}}=\mathbf{W}_{\mathrm{k}}^{\mathrm{T}} \boldsymbol{\varphi}_{\mathrm{k}}
$$

$\mathbf{T}_{\mathrm{k}}$ is a two-scale transform between the scales k-1 and k, such that

$$
\left\{\begin{array}{c}
\varphi_{k-1} \\
\psi_{k}
\end{array}\right\}=\mathbf{T}_{\mathrm{k}}^{\mathrm{T}} \varphi_{\mathrm{k}}
$$

with

$$
\psi_{\mathrm{k}}^{\mathrm{j}}=\varphi_{\mathrm{k}}^{2 \mathrm{j}-1}, \mathrm{j}=1, \ldots, \mathrm{N}_{\mathrm{k}}
$$

$\mathrm{N}_{\mathrm{k}}$ denotes here the total number of the FEM nodal points at the scale k. Let us illustrate the wavelet-based FEM idea using the example of 1D linear two-noded finite element with the shape functions [9]

$$
\mathbf{N}^{\mathrm{T}}=\left\{\begin{array}{l}
\mathrm{N}_{1} \\
\mathrm{~N}_{2}
\end{array}\right\}=\left\{\begin{array}{l}
\frac{1}{2}(1-\xi) \\
\frac{1}{2}(1+\xi)
\end{array}\right\},
$$

where $\mathrm{N}_{1}$ is valid for $\xi=-1$ and $\mathrm{N}_{2}$ - for $\xi=1$ in local coordinates system of this element. The scale effect is introduced on the element level by inserting new extra degrees of freedom at each new scale. Then, the scale 1 corresponds to first extra multiscale DOF per the original finite element, scale 2 - next two additional multiscale DOFs and etc. It may be generally characterized as

where

$$
\psi_{k}(\xi)=\psi_{k}\left(2^{k-1}(1+\xi)-2 j-1\right)
$$

$$
\left\{\begin{array}{c}
2^{2-k} j-1 \leq \xi \leq 2^{2-k} j+2^{1-k}-1 \\
2^{2-k} j+2^{1-k}-1 \leq \xi \leq 2^{2-k} j+2^{2-k}-1
\end{array}\right.
$$

The value of $\mathrm{k}$ defines the actual scale. The reconstruction algorithm starts from the original solution for the original mesh. Next, the new scales are introduced using the formula

$$
\mathbf{u}_{k}^{2+2^{k-1}+j}=\sum_{i=1}^{N_{\text {old }}} \mathbf{N}_{i} \mathbf{u}_{0}^{i}+\sum_{i=1}^{N_{\text {new }}} \psi_{k}^{2+2^{k-1}+j} \Delta \mathbf{u}_{k}^{2+2^{k-1}+j} .
$$

The wavelet algorithm for stiffness matrix reconstruction starts at scale 0 with the smallest rank stiffness matrix

$$
\mathbf{K}_{0}=\frac{\mathrm{e}}{\mathrm{h}}\left[\begin{array}{cc}
1 & -1 \\
-1 & 1
\end{array}\right],
$$

where $\mathrm{h}$ is the node spacing parameter. Then, the diagonal components of the stiffness matrix for any $\mathrm{k}>0$ are equal to 


$$
\mathrm{K}_{\mathrm{k}}^{2+2^{\mathrm{K}-1}+\mathrm{J}}=\frac{2^{\mathrm{k}+1} \mathrm{e}}{\mathrm{h}} .
$$

It should be underlined that the FEM so modified reflects perfectly the needs of computational modeling of multiscale heterogeneous media. The reconstruction algorithm can be applied for such $\mathrm{n}$, which assure a sufficient mesh zoom on the smallest scale in the composite.

\section{Finite Element Method Equations of the Problem}

The following variational equation is proposed to study dynamic equilibrium for the linear elastic system:

$$
\int_{\Omega} \rho \ddot{u}_{\mathrm{i}} \delta \mathrm{u}_{\mathrm{i}} \mathrm{d} \Omega+\int_{\Omega} \mathrm{C}_{\mathrm{ijkl}} \varepsilon_{\mathrm{ij}} \delta \varepsilon_{\mathrm{kl}} \mathrm{d} \Omega=\int_{\Omega} \rho \mathrm{f}_{\mathrm{i}} \delta \mathrm{u}_{\mathrm{i}} \mathrm{d} \Omega+\int_{\partial \Omega_{\sigma}} \hat{\mathrm{t}}_{\mathrm{i}} \delta \mathrm{u}_{\mathrm{i}} \mathrm{d}(\partial \Omega)
$$

and $\mathrm{u}_{\mathrm{i}}$ represents displacements of the system $\Omega$ with elastic properties and mass density defined by the elasticity tensor $\mathrm{C}_{\mathrm{ijkl}}(\mathrm{x})$ and $\rho=\rho(\mathbf{x})$; the vector $\hat{\mathrm{t}}_{\mathrm{i}}$ denotes the stress boundary conditions imposed on $\partial \Omega_{\sigma} \subset \partial \Omega$. Analogous equation for the homogenized medium has the following form:

$$
\int_{\Omega} \rho^{(\text {eff })} \ddot{u}_{\mathrm{i}} \delta \mathrm{u}_{\mathrm{i}} \mathrm{d} \Omega+\int_{\Omega} \mathrm{C}_{\mathrm{ijkl}}^{(\text {eff })} \varepsilon_{\mathrm{ij}} \delta \varepsilon_{\mathrm{kl}} \mathrm{d} \Omega=\int_{\Omega} \rho^{(\text {eff })} \mathrm{f}_{\mathrm{i}} \delta \mathrm{u}_{\mathrm{i}} \mathrm{d} \Omega+\int_{\partial \Omega} \mathrm{t}_{\mathrm{i}} \delta \mathrm{u}_{\mathrm{i}} \mathrm{d}(\partial \Omega)
$$

where all material properties of the real system are replaced with the effective parameters. As it is known [9], classical FEM discretization returns the following equations for real heterogeneous and homogenized systems are obtained:

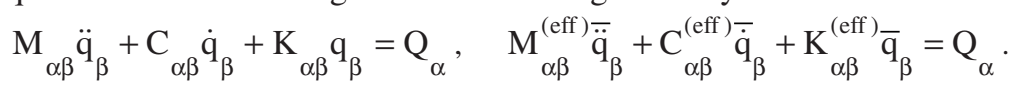

The R.H.S. vector equals to 0 for free vibrations and then an eigenvalue problem is solved using the following matrix equations:

$$
\left(\mathrm{K}_{\alpha \beta}-\omega_{(\alpha)} \mathrm{M}_{\alpha \beta}\right) \Phi_{\beta \gamma}=0, \quad\left(\mathrm{~K}_{\alpha \beta}^{(\mathrm{eff})}-\bar{\omega}_{(\alpha)} \mathrm{M}_{\alpha \beta}^{(\mathrm{eff})}\right) \bar{\Phi}_{\beta \gamma}=0 .
$$

\section{Computational Illustration}

First, simply supported periodic composite beam is analyzed, where Young modulus $\mathrm{E}(\mathrm{x})$ and mass density in the periodicity cell are given by the following wavelets:

$$
\begin{gathered}
h(x)=\left\{\begin{array}{c}
20.0 \mathrm{E} 9 ; 0 \leq \mathrm{x} \leq 0.5 \\
2.0 \mathrm{E} 90.5<\mathrm{x} \leq 1
\end{array}, \quad \mathrm{~m}(\mathrm{x})=2+\frac{1}{\sqrt{2 \pi} \sigma^{3}} \frac{\mathrm{x}^{2}}{\sigma^{2}-1} \exp \left(\frac{-\mathrm{x}^{2}}{2 \sigma^{2}}\right), \sigma=-0.4 ;\right. \\
\mathrm{E}(\mathrm{x})=10.0 \cdot \mathrm{h}(\mathrm{x})+2.0 \mathrm{E} 9 \cdot \mathrm{m}(\mathrm{x}) . \\
\tilde{\mathrm{h}}(\mathrm{x})=\left\{\begin{array}{c}
200 ; 0 \leq \mathrm{x} \leq 0.5 \\
20 ; 0.5<\mathrm{x} \leq 1
\end{array}, \quad \rho(\mathrm{x})=0.5 \cdot \tilde{\mathrm{h}}(\mathrm{x})+0.5 \cdot \mathrm{m}(\mathrm{x}) .\right.
\end{gathered}
$$


The composite specimen is discretized using each time 128 2-noded linear finite elements with unitary inertia moments. The comparison starts from a collection of the eigenvalues reflecting different homogenization techniques given in Tab. 1. Further, the eigenvalues for heterogeneous beams are given for $1^{\text {st }}$ order wavelet projection in Tab. 2, for $2^{\text {nd }}$ order projection - in Tab. $3,3^{\text {rd }}$ order - in Tab. 4.

The eigenvalues computed for various homogenization models approximate the values computed for the real composite models with different accuracy - the weakest efficiency is detected in case of spatially averaged composite and the difference in relation to the real structure results increase together with the eigenvalue number and the projections order. The results obtained thanks to MRA projection are closer to those relevant to MRA homogenization for a single RVE in composite; classical homogenization is more effective for increasing number of the cells in this model.

Table 1. Eigenvalues for the simply supported homogenized composite beams

\begin{tabular}{cccc}
\hline Eigenvalue & Spatial averaging & Classical approach & Multiresolutional model \\
\hline 1 & $1,184 \mathrm{E} 12$ & $3,665 \mathrm{E} 11$ & $6,228 \mathrm{E} 11$ \\
2 & $1,895 \mathrm{E} 13$ & $5,864 \mathrm{E} 12$ & $9,965 \mathrm{E} 12$ \\
3 & $9,592 \mathrm{E} 13$ & $2,969 \mathrm{E} 13$ & $5,045 \mathrm{E} 13$ \\
4 & $3,032 \mathrm{E} 14$ & $9,383 \mathrm{E} 13$ & $1,594 \mathrm{E} 14$ \\
5 & $7,401 \mathrm{E} 14$ & $2,291 \mathrm{E} 14$ & $3,893 \mathrm{E} 14$ \\
6 & $1,535 \mathrm{E} 15$ & $4,750 \mathrm{E} 14$ & $8,072 \mathrm{E} 14$ \\
\hline
\end{tabular}

Table 2. Eigenvalues for the simply supported composite beam, $1^{\text {st }}$ order wavelet projection

\begin{tabular}{ccccccc}
\hline 64 RVEs & 32 RVEs & 16 RVEs & 8 RVEs & 4 RVEs & 2 RVEs & 1 RVE \\
\hline 3,534 E11 & $3,535 \mathrm{E} 11$ & $3,537 \mathrm{E} 11$ & $3,550 \mathrm{E} 11$ & $3,599 \mathrm{E} 11$ & $3,829 \mathrm{E} 11$ & $4,529 \mathrm{E} 11$ \\
$5,656 \mathrm{E} 12$ & $5,660 \mathrm{E} 12$ & $5,679 \mathrm{E} 12$ & $5,760 \mathrm{E} 12$ & $6,137 \mathrm{E} 12$ & $7,887 \mathrm{E} 12$ & $2,593 \mathrm{E} 13$ \\
$2,864 \mathrm{E} 13$ & $2,870 \mathrm{E} 13$ & $2,892 \mathrm{E} 13$ & $2,991 \mathrm{E} 13$ & $3,512 \mathrm{E} 13$ & $4,973 \mathrm{E} 13$ & $7,317 \mathrm{E} 13$ \\
$9,056 \mathrm{E} 13$ & $9,087 \mathrm{E} 13$ & $9,216 \mathrm{E} 13$ & $9,828 \mathrm{E} 13$ & $1,315 \mathrm{E} 14$ & $4,867 \mathrm{E} 14$ & $3,512 \mathrm{E} 14$ \\
$2,212 \mathrm{E} 14$ & $2,224 \mathrm{E} 14$ & $2,275 \mathrm{E} 14$ & $2,536 \mathrm{E} 14$ & $3,758 \mathrm{E} 14$ & $6,743 \mathrm{E} 14$ & $6,241 \mathrm{E} 14$ \\
$4,591 \mathrm{E} 14$ & $4,627 \mathrm{E} 14$ & $4,786 \mathrm{E} 14$ & $5,655 \mathrm{E} 14$ & $8,448 \mathrm{E} 14$ & $1,347 \mathrm{E} 15$ & $1,678 \mathrm{E} 15$ \\
\hline
\end{tabular}

Table 3. Eigenvalues for the simply supported composite beam, $2^{\text {nd }}$ order wavelet projection

\begin{tabular}{cccccc}
\hline 32 RVEs & 16 RVEs & 8 RVEs & 4 RVEs & 2 RVEs & 1 RVE \\
\hline 3,636 E11 & $3,639 \mathrm{E} 11$ & $3,652 \mathrm{E} 11$ & $3,703 \mathrm{E} 11$ & $3,936 \mathrm{E} 11$ & $4,604 \mathrm{E} 11$ \\
$5,823 \mathrm{E} 12$ & $5,842 \mathrm{E} 12$ & $5,925 \mathrm{E} 12$ & $6,309 \mathrm{E} 12$ & $8,006 \mathrm{E} 12$ & $2,603 \mathrm{E} 13$ \\
$2,952 \mathrm{E} 13$ & $2,975 \mathrm{E} 13$ & $3,075 \mathrm{E} 13$ & $3,605 \mathrm{E} 13$ & $5,090 \mathrm{E} 13$ & $7,420 \mathrm{E} 13$ \\
$9,348 \mathrm{E} 13$ & $9,480 \mathrm{E} 13$ & $1,010 \mathrm{E} 14$ & $1,334 \mathrm{E} 14$ & $4,875 \mathrm{E} 14$ & $3,531 \mathrm{E} 14$ \\
$2,288 \mathrm{E} 14$ & $2,340 \mathrm{E} 14$ & $2,605 \mathrm{E} 14$ & $3,846 \mathrm{E} 14$ & $6,803 \mathrm{E} 14$ & $6,292 \mathrm{E} 14$ \\
$4,760 \mathrm{E} 14$ & $4,921 \mathrm{E} 14$ & $5,805 \mathrm{E} 14$ & $8,641 \mathrm{E} 14$ & $1,362 \mathrm{E} 15$ & $1,690 \mathrm{E} 15$ \\
\hline
\end{tabular}

Table 4. Eigenvalues for the simply supported composite beam, $3^{\text {rd }}$ order wavelet projection

\begin{tabular}{|c|c|c|c|c|}
\hline 16 RVEs & 8 RVEs & 4 RVEs & 2 RVEs & $1 \mathrm{RVE}$ \\
\hline $3,662 \mathrm{E} 11$ & $3,674 \mathrm{E} 11$ & $3,726 \mathrm{E} 11$ & 3,964 E11 & $4,664 \mathrm{E} 11$ \\
\hline 5,879 E12 & 5,962 E12 & $6,354 \mathrm{E} 12$ & $8,121 \mathrm{E} 12$ & $2,600 \mathrm{E} 13$ \\
\hline 2,993 E13 & $3,096 \mathrm{E} 13$ & 3,637 E13 & 5,174 E13 & 7,479 E13 \\
\hline 9,540 E13 & 1,017 E14 & 1,354 E14 & $4,876 \mathrm{E} 14$ & $3,529 \mathrm{E} 14$ \\
\hline 2,355 E14 & $2,626 \mathrm{E} 14$ & 3,903 E14 & $6,839 \mathrm{E} 14$ & $6,341 \mathrm{E} 14$ \\
\hline 4,954 E14 & 5,857 E14 & 8,796 E14 & 1,373 E15 & $1,691 \mathrm{E} 15$ \\
\hline
\end{tabular}


Free vibrations for 2 and 3-bays periodic beams are solved using classical and homogenization-based FEM implementation. The unitary inertia momentum is taken in all computational cases, ten periodicity cells compose each bay, while material properties inserted in the numerical model are calculated by spatial averaging, classical and multiresolutional homogenization schemes and compared against the real structure response. First 10 eigenvalues changes for all these beams are contained in Figs. 1,2 - the resulting values are marked on the vertical axes, while the number of eigenvalue being computed - on the horizontal ones.

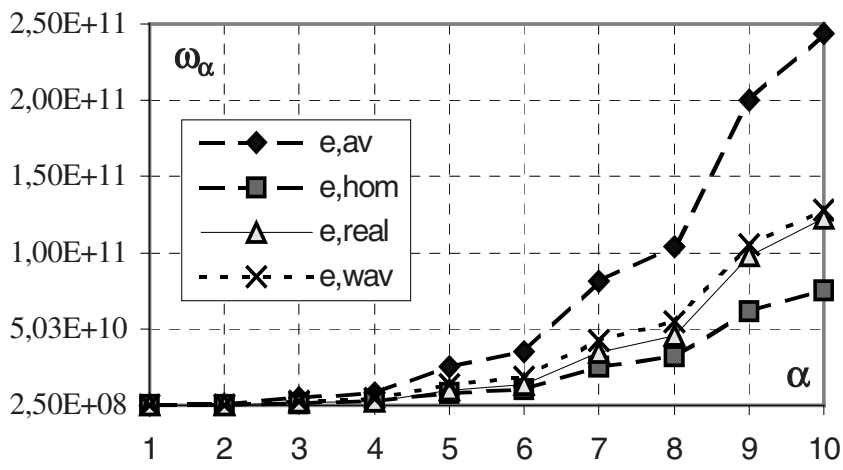

Fig. 1. Eigenvalues progress for various two-bays composite structures

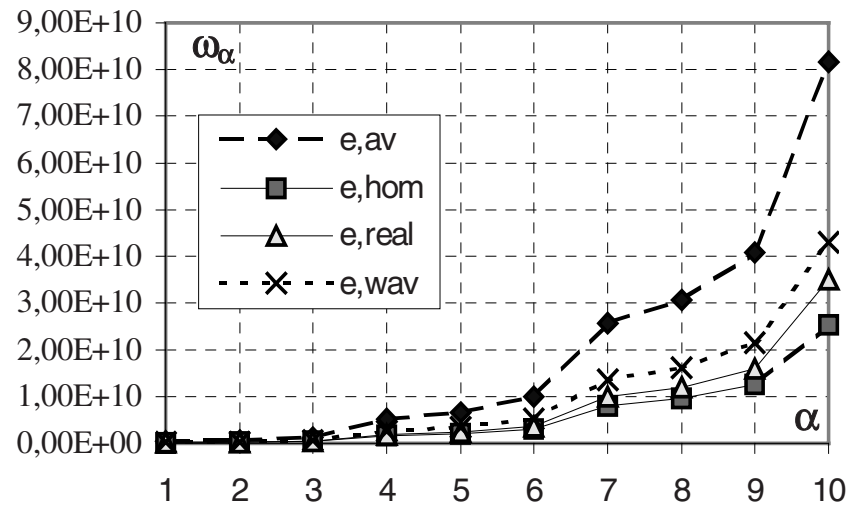

Fig. 2. Eigenvalues progress for various three-bays composite structures

Eigenvalues obtained for various homogenization models approximate the values computed for the real composite with different accuracy - the worst efficiency in eigenvalues modeling is detected in case of spatially averaged composite and the difference in relation to the real structure results increase together with the eigenvalue number. Wavelet-based and classical homogenization methods give more accurate results - the first method is better for smaller number of the bays, and classical homogenization approach is recommended in case of increasing number of the bays and the RVEs. The justification of this observation comes from the fact, that the wavelet function is less important for the increasing number of the periodicity cells in 
the structure. Another interesting result is that the efficiency of approximation of the maximum deflections for a multi-bay periodic composite beam by the deflections encountered for homogenized systems increase together with an increase of the total number of the bays.

\section{Conclusions}

The most important result of the homogenization-based Finite Element modeling of the periodic unidirectional composites is that the real composite behavior is rather well approximated by the homogenized model response. MRA homogenization technique giving more accurate approximation of the real structure behavior is decisively more complicated in numerical implementation since necessity of usage of the combined symbolic-FEM approach. The technique introduces new opportunities to calculate effective parameters for the composites with material properties approximated by various wavelet functions. A satisfactory agreement between the real and homogenized structures models enables the application to other transient problems with deterministic as well as stochastic material parameters.

Multiresolutional homogenization procedure has been established here using the Haar basis to determine complete mathematical equations for homogenized coefficients and to make implementation of the FEM-based homogenization analysis. As it was documented above, the Haar basis approximation gives sufficient approximation of various mathematical functions describing most of possible spatial distributions of composites physical properties.

\section{References}

1. Al-Aghbari, M., Scarpa, F., Staszewski, W.J., On the orthogonal wavelet transform for model reduction/synthesis of structures. J. Sound \& Vibr. 254(4), pp. 805-817, 2002.

2. Christon, M.A. and Roach, D.W., The numerical performance of wavelets for PDEs: the multi-scale finite element, Comput. Mech., 25, pp. 230-244, 2000.

3. Dorobantu M., Engquist B., Wavelet-based numerical homogenization, SIAM J. Numer. Anal., 35(2), pp. 540-559, 1998.

4. Gilbert, A.C., A comparison of multiresolution and classical one-dimensional homogenization schemes, Appl. \& Comput. Harmonic Anal., 5, pp. 1-35, 1998.

5. Kamiński, M., Multiresolutional homogenization technique in transient heat transfer for unidirectional composites, Proc. $3^{\text {rd }}$ Int. Conf. Engineering Computational Technology, Topping, B.H.V. and Bittnar Z., Eds, Civil-Comp Press, 2002.

6. Kamiński, M., Wavelet-based finite element elastodynamic analysis of composite beams, WCCM V, Mang, H.A., Rammerstorfer, F.G. and Eberhardsteiner, J., Eds, Vienna 2002.

7. Sanchez-Palencia, E., Non-homogeneous Media and Vibration Theory. Lecture Notes in Physics, vol. 127, Springer-Verlag, Berlin, 1980.

8. Steinberg, B.Z. and McCoy, J.J., A multiresolution homogenization of modal analysis with application to layered media, Math. Comput. \& Simul., 50, pp. 393-417, 1999.

9. Zienkiewicz, O.C. and Taylor, R.L., The Finite Element Method. Heinemann-Butterworth, 2000. 